\title{
Antenatal diagnosis of large sacro-coccygeal teratoma with foetal cardiomegaly and hydrops
}

\author{
Gupta $\mathbf{N}^{1}$, Shah $\mathrm{D}^{2}$, Singh $\mathrm{U}^{3}$, Tiwari $\mathrm{A}^{1}$
}

${ }^{1}$ Assistant Professor, Departments of Radiology, ${ }^{2}$ Associate Professor, Departments of Paediatrics, ${ }^{3}$ Professor, Departments of Obstetrics \& Gynaecology, B.P. Koirala Institute of Health Sciences, Dharan, Nepal

\begin{abstract}
We report the case of a young primigravida who presented with a 20 weeks pregnancy with a previous diagnosis of uterine fibroid. However, ultrasound evaluation revealed the presence of a large sacro-coccygeal teratoma in the foetus which was heterogeneous with cystic areas and calcific foci, and showed increased vascularity. There was also evidence of foetal cardiomegaly and hydrops foetalis, indicated by subcutaneous oedema in the foetus, thickened placenta and polyhydramnios. The presence of hydrops with sacro-coccygeal teratoma has a grave prognosis for the mother and child; hence termination of pregnancy was done. A 20 weeks old foetus with a huge sacro-coccygeal mass, which was ruptured at many places and showed areas of active bleed, was expelled.
\end{abstract}

\begin{abstract}
acro-coccygeal teratoma (SCT) is the most common of all foetal neoplasms. Large, solid and vascular tumours can cause complications such as cardiomegaly and hydrops in the foetus secondary to arterio-venous shunting. These are associated with a poor outcome for both the baby and the mother. Therefore, it is important to make a correct and timely antenatal diagnosis of the condition and follow it up for complications, so that appropriate action can be taken.
\end{abstract}

\section{Case report}

We report the case of a 23 year old primigravida who came to our department for antenatal ultrasound evaluation with history of amenorrhoea for 20 weeks. One ultrasound examination had been done about 2 weeks earlier at a private centre which reported the presence of a uterine fibroid co-existing with the pregnancy. The mother was otherwise asymptomatic and did not have any complaints.

The ultrasound done in our centre revealed a viable intrauterine foetus with femur length corresponding to 21 weeks of gestation. There was a huge heterogeneous mass lesion seen arising from the sacro-coccygeal region of the foetus, showing solid and cystic areas, foci of calcification (Fig 1) and increased vascularity on doppler evaluation. The major part of the mass was external to the foetus, situated posteriorly and caudally, with a small component seen extending in the foetal pelvis anteriorly (Fig 2). The mass was very large, measuring about $12 \times 12.5 \times 11 \mathrm{~cm}$ on antenatal imaging.
There was also evidence of hypoechoic subcutaneous thickening in the head, chest and abdomen of foetus, diagnosed as subcutaneous oedema. The heart was significantly enlarged, occupying a large part of chest, with small sized lungs, suggestive of pulmonary hypoplasia. Amniotic fluid was seen to be increased in volume with amniotic fluid index (AFI) of 26 and also, there was evidence of placentomegaly with a transverse placental diameter of about $6 \mathrm{~cm}$. The final ultrasound diagnosis was a large foetal sacro-coccygeal teratoma with foetal cardiomegaly and hydrops (Fig 3). No evidence of any uterine lesion was found in the scan.

The parents decided to terminate the pregnancy after appropriate explanation of prognosis, in conjunction with the department of Obstetrics and Gynaecology. Vaginal delivery was conducted with expulsion of a female foetus with a huge, lobulated, vascular sacrococcygeal mass which had ruptured at places during the delivery and showed areas of active haemorrhage (Fig 4). Significant subcutaneous oedema was seen in the foetus secondary to hydrops. The parents did not give consent for foetal autopsy. 


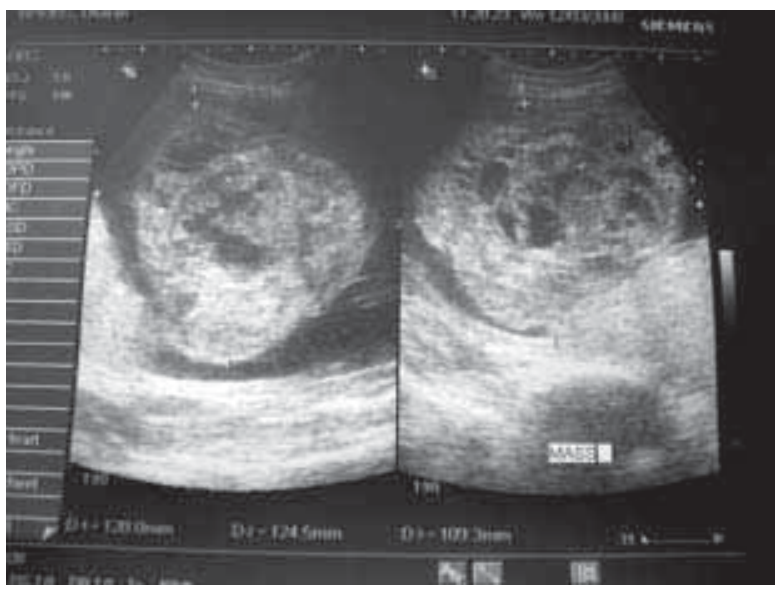

Fig 1: Large heterogeneous mass with multiple cystic ares and small calcific foci.

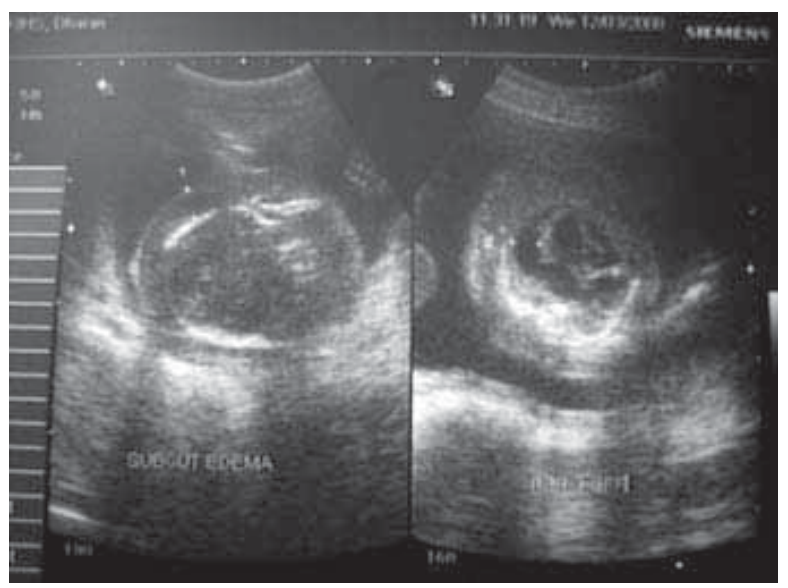

Fig 3: a) Subcutaneous edema in fetal abdomen.

b) Fetal cardiomegaly with pulmonary hypoplasia.

\section{Discussion}

Foetal tumours form a heterogeneous group which differs widely in their histology, pathophysiology, location and biologic behaviour. Of these, teratomas are the most frequent histologic subtype ${ }^{1}$. Teratomas arise from pleuripotent germ cells that may be arrested at various midline locations, during their migration to the genital ridges in intrauterine life. They are composed of all three germ layers- ectoderm, mesoderm and endoderm. Ectodermal elements- particularly neural tissue forms the major component, followed by mesodermal elements like fat, bone, smooth muscle and cartilage. Endodermal components as respiratory and gastro-intestinal tissues may also be seen less frequently. Teratomas may be found in any midline location from the pineal gland to coccyx- in order of frequency- the sacro-coccygeal region, head and neck, anterior mediastinum and the retroperitoneum.

Sacro-coccygeal teratomas are the commonest foetal and neonatal neoplasms with an incidence of 1 in 30-

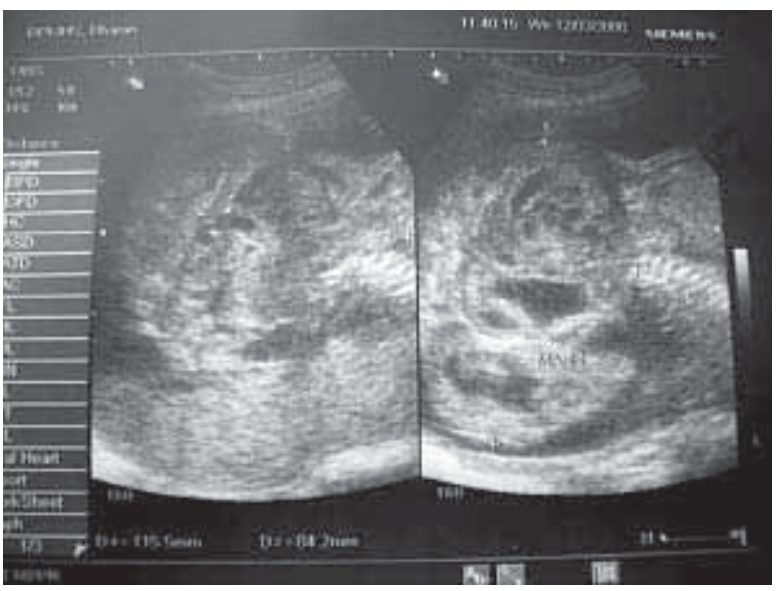

Fig 2: Sacrococcygeal tumour with large external and small presacral component

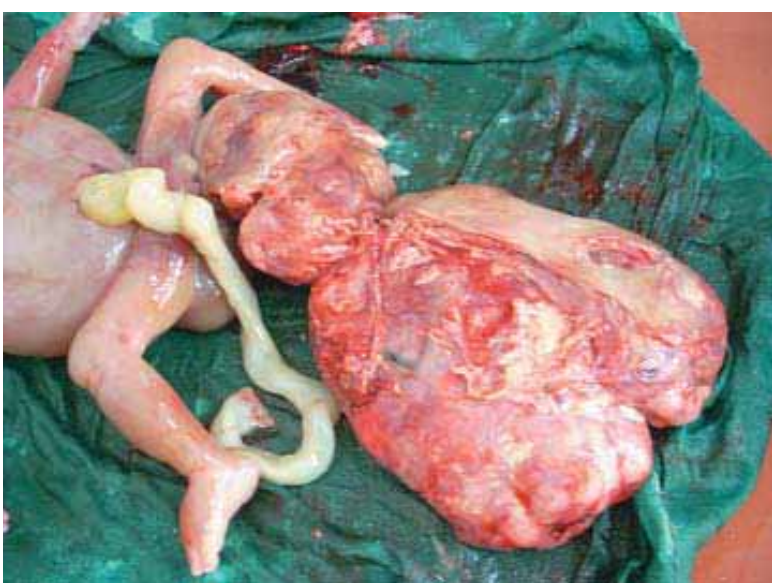

Fig 4: Fetus showing hydrops and large, lobulated sacro-coccygeal mass with areas of rupture and active hemorrhage.

40,000 live births and a male: female ratio of 1:4. It is important to accurately diagnose SCT antenatally as an undiagnosed or misdiagnosed tumour may result in a grave prognosis for the foetus as well as the mother. The most important sonographic criteria which portend an unfavourable outcome are foetal cardiomegaly, hydrops foetalis and increased preload indices on the foetal venous system signifying foetal cardiac failure (indicated by increased inferior venacaval diameter and pulsatile flow in umbilical vein on Doppler evaluation $)^{2}$. Acute haemorrhage has also been seen into the tumour resulting in foetal anemia ${ }^{3}$. If the above complications develop before 37 weeks, foetal mortality is $100 \%{ }^{4}$. The maternal risk results from pre- eclampsia and respiratory insufficiency, related to increased beta-HCG levels ${ }^{5}$. The maternal 'mirror syndrome' has been described, a life-threatening condition with maternal fluid retention and hemodilution, which occurs with foetal hydrops, and is seen as increasing maternal oedema "mirroring" that of the affected foetus ${ }^{4}$. In the absence 
of any complications, delivery by caesarean section at optimum foetal maturity, followed by surgical removal of tumour has a good prognosis.

The typical imaging finding in SCT is a heterogeneous, mixed solid and cystic mass lesion, which may exhibit areas of calcification. Increased vascularity may be seen on Doppler evaluation. Up to $15 \%$ tumours can be entirely cystic and have a better prognosis. The mass may be totally external to the foetus, show intrapelvic extension, or be entirely internal in location. Magnetic Resonance Imaging (MRI) is a better modality to assess for intrapelvic extension. The lesion may also less commonly extend into the spinal canal. The American Academy of Paediatrics Surgery Section Survey (AAPSSS) has classified SCT into four types based on the internal and external location of the mass ${ }^{1}$.

Type I - the mass is external with minimal or no internal components.

Type II - the mass is predominantly external, with internal extension into presacral space.

Type III - mass is both external and internal with extension into the abdominal cavity.

Type IV - mass is entirely internal with no external component

These tumours can grow very fast, reaching huge volumes and can even outgrow the foetus in size. Large solid tumours with a high vascularity, secondary to increased metabolic demand and arterio-venous shunting causing vascular steal, can result in high output cardiac failure and hydrops foetalis ${ }^{1}$. The earliest signs of hydrops in the foetus are polyhydramnios and placentomegaly ${ }^{4}$. If hydrops develops, then the prognosis is invariably poor.

Polyhydramnios is frequently seen with SCT. Oligohydramnios is less common. Pulmonary hypoplasia may occur secondary to oligohydramnios or chronic cardiomegaly. Other associated foetal anomalies may be hydronephrotic or dysplastic kidneys, urethral/ rectal atresias, hip dislocation, and clubfeet etc ${ }^{1}$.

The main differential diagnosis for a primarily cystic tumour is meningomyelocele, which is seen as a cystic mass in the lumbo-sacral region, associated with spinal dysraphism. Chordoma, neuroblastoma and neurenteric cyst form the other differentials for a predominantly internal variety of teratoma.
Our case showed the typical imaging features of SCTa large, heterogenous, solid-cystic, vascular mass with internal calcific foci. It was a large external mass with a smaller internal extension, so was classified as type II according to the AAPSSS system of classification. The lesion showed increased vascularity, with presence of foetal cardiomegaly, hydrops and pulmonary hypoplasia already at the time of diagnosis, hence had a poor prognosis and so, termination of pregnancy was carried out. The mother, although at the time of referral, was asymptomatic, however, carried a real risk of developing serious complications as described above, in the event of continuation of pregnancy after the previously missed diagnosis.

The final message is that SCT, being the commonest foetal tumour, may not be infrequently encountered in practice, and in the event of developing complications, may prove to be potentially catastrophic for both the mother and foetus. It is therefore, essential, that an accurate timely diagnosis bemade, (forwhich sonography is a simple, low cost and readily available modality), adequate surveillance done for any complications and appropriate interventions carried out.

\section{References}

1. Woodward PJ, Sohaey R, Kennedy A, Koeller KK. A comprehensive review of foetal tumours with pathologic correlation. Radiographics. 2005; 25: 215-42.

2. Neubert S, Trautmann K, Tanner B, Steiner E, Linke F, Bahlmann F. Sonographic prognostic factors in prenatal diagnosis of SCT. Foetal Diagn Ther 2004; 19:319-26.

3. Yamaguchi Y, Tsukimori K, Hojo S, Nakanami $\mathrm{N}$, Nozaki M, Masumoto $\mathrm{K}$, Taguchi $\mathrm{T}$. Spontaneous rupture of sacro-coccygeal teratoma associated with acute foetal anemia. Ultrasound Obstet Gynecol. 2006; 28; 720-2.

4. Makin EC, Hyett J, Ade-Ajayi N, Patel S, Nicolaides K, Davenport M. Outcome of antenatally diagnosed sacrococcygeal teratomas: single-center experience (1993-2004). J Pediatr Surg. 2006; 41: 388-93.

5. Bock B, Riess R, Wünsch PH, Feige A. Prenatal diagnosis of a sacrococcygeal teratoma with hydrops foetalis and placental hypertrophy-consequences for the further course of pregnancy. Geburtshilfe Frauenheilkd. 1990; 50: 647-9. 